\title{
Issue procedure ontology (ipo): uma ontologia para sintomas, problemas e soluções
}

Matheus Dimas de Morais

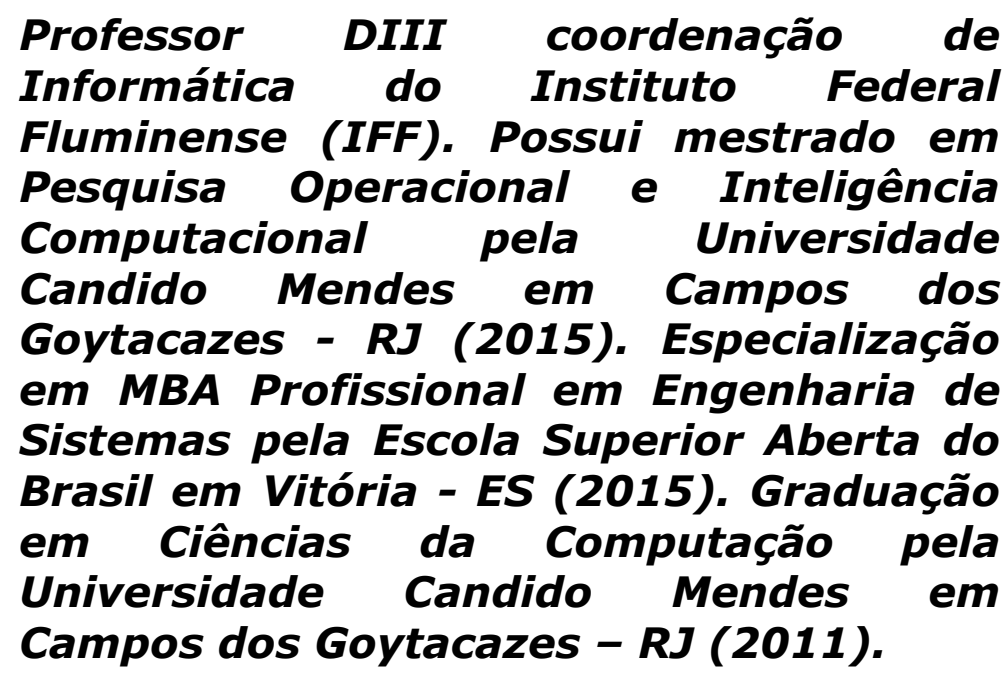

Mark Douglas de Azevedo Jacyntho

Professor adjunto da coordenação de Ciência da Computação, da Universidade Candido Mendes em Campos dos Goytacazes - RJ. Professor DIV da coordenação de Informática do Instituto Federal Fluminense (IFF). Possui doutorado em Informática (2012), mestrado em Informática (2002) e graduação em Engenharia de Computação (1998), os três títulos obtidos no Departamento de Informática da Pontifícia Universidade Católica do Rio de Janeiro (PUC-Rio).

http://dx.doi.org/10.1590/1981-5344/2446

No cenário atual de intensa competitividade entre as empresas, cada vez mais o uso do tempo se torna algo a ser maximizado, visando maior produtividade. Assim, para as organizações se manterem em plena operação, problemas devem ser solucionados o mais breve possível. A informática pode ajudar nesse processo, oferecendo sistemas computacionais que auxiliem na resolução de tais problemas. Com o advento da Web Semântica, surge 
a possibilidade de representar as informações de tal forma que o computador consiga compreendê-las, possibilitando a criação de sistemas inteligentes com o uso de ontologias. A principal contribuição deste trabalho é uma ontologia extensível (core ontology) para o domínio de sintomas, problemas e soluções, denominada Issue Procedure Ontology (IPO). Esta ontologia pretende prover às máquinas a semântica necessária para que estas forneçam não apenas informações, mas, sobretudo, identifiquem problemas a partir de um conjunto de sintomas e, em seguida, sugiram possíveis soluções, de forma autônoma, para os problemas em questão. Ao final, é realizada uma avaliação da ontologia a fim de corroborar seu poder de expressividade.

Palavras-chave: Ontologia extensível. Sintomas, problemas e soluções. Web semântica.

\section{Issue procedure ontology (ipo): an ontology for symptoms, problems and solutions}

In the current scenario of intense competitiveness among companies, use of time increasingly becomes something to be maximized, aiming at greater productivity. Thus, for organizations remain in full operation, problems should be solved as soon as possible. Information technology can help this process by providing computer systems to assist in troubleshooting. With the advent of the Semantic Web, there is the possibility to represent information in such a way that the computer can understand them, enabling the creation of intelligent systems with the use of ontologies. The main contribution of this work is an extensible ontology (core ontology) for the domain of symptoms, problems and solutions, named Issue Procedure Ontology (IPO). This ontology aims to provide the necessary semantics for machines so that they provide not only information, but, above all, identify problems from a set of symptoms and then autonomously suggest possible solutions for the problems in question. In the end, is performed an evaluation of ontology in order to corroborate its expressiveness.

Keywords: Core ontology. Symptoms, problems and solutions. Semantic Web. 


\section{Introdução}

No cenário atual de intensa competitividade entre as empresas, cada vez mais o uso do tempo se torna algo a ser maximizado, visando maior produtividade. Assim, as organizações devem alocar seus recursos e gerenciá-los de forma adequada, para que a força de trabalho tenha plena condição de realizar suas tarefas com maior eficiência (MAGALHÃES; PINHEIRO, 2007; REZENDE, 2002).

A área de Tecnologia da Informação (TI) tem sido de suma importância para as organizações, uma vez que os sistemas computacionais podem agilizar o trabalho feito por seus colaboradores, servindo como meio para as organizações alcançarem seus objetivos. Hoje, para muitas empresas, a TI se tornou parceira estratégica, fazendo parte do negócio da empresa (PEREIRA; DE SOUZA; DA COSTA, 2012).

Assim, os serviços de TI podem ajudar diversos setores das empresas a solucionar seus problemas. Um problema geralmente possui sintomas que indicam sua existência e uma ou mais possíveis soluções, meios que se consiga sanar (ou amenizar) o problema, eliminando (ou reduzindo), assim, os sintomas.

O processo de (i) analisar os sintomas, (ii) identificar o problema e (iii) localizar a solução, depende da ação humana do profissional, que pode errar em seu diagnóstico, devido sua falta de experiência ou atenção. Desta forma, sistemas computacionais podem ser utilizados para facilitar e auxiliar o profissional nessa importante tarefa.

Com o advento da Web Semântica ou Web de Dados Ligados (Web of Linked Data), surge a possibilidade de representar as informações de maneira que o computador consiga compreendê-las (BERNERS-LEE; HENDLER; LASSILA, 2001).

Para que os dados sejam compreendidos pelos computadores, se faz necessário que os mesmos sejam estruturados de forma padronizada e que seja dado sentido semântico explícito a eles. Para associar semântica aos dados, o consórcio $\mathrm{W} 3 \mathrm{C}^{1}$ propõe a utilização de ontologias ou vocabulários. Ontologia é uma representação formal de um determinado domínio de conhecimento, onde conceitos (classes) deste domínio são explicitamente definidos, bem como suas propriedades e relacionamentos (JACYNTHO, 2012). São as ontologias que dão poder de inferência às máquinas, possibilitando, com uso de raciocinadores (softwares que fazem uso dos axiomas ontológicos para inferir novos dados), a descoberta de novos conhecimentos (LICHTNOW; DE OLIVEIRA, 2009; PICKLER, 2007).

Visando prover uma representação semântica para o domínio de sintomas, problemas e soluções, foi projetada uma ontologia extensível (core ontology) denominada Issue Procedure Ontology (IPO) que pretende prover às máquinas a semântica necessária para que estas forneçam não

\footnotetext{
${ }^{1}$ Disponível em: <http://www.w3.org/>. Acesso em: 15 maio 2015.
} 
apenas informações, mas, sobretudo, identifiquem problemas a partir de um conjunto de sintomas e, em seguida, sugiram possíveis soluções, de forma autônoma, para os problemas em questão. A intenção é prover uma ontologia genérica, reutilizável e extensível para o domínio de sintomasproblemas-soluções. Uma ontologia que pode ser usada diretamente, mas que também pode ser estendida ou especializada para tipos de problemas mais específicos.

Este artigo tem como objetivo apresentar a ontologia IPO. Para tal, foi organizado da seguinte forma: seção 2 expõe, brevemente, 0 referencial teórico sobre ontologia e o domínio de problemas; na seção 3 é apresentada a ontologia em si, abordando os passos metodológicos e suas classes e propriedades; seção 4 apresenta um exemplo de instanciação da ontologia; para complementar, na seção 5, é demonstrado um procedimento, com o detalhamento de todos os seus passos (workflow); já a seção 6 é dedicada a uma avaliação da ontologia, com base nos exemplos anteriores, mostrando que a mesma permite, de fato, responder as questões de competências que motivaram a sua criação; finalmente, na seção 7, este trabalho é concluído com as observações finais e trabalhos futuros.

\section{Referencial teórico}

\subsection{Web semântica e ontologias}

A Web Semântica pretende evoluir a Web atual, conhecida como "Web de documentos", que tem como principal objetivo disponibilizar informações para as pessoas, para a "Web de dados" que visa disponibilizar os dados para que as máquinas possam manipulá-los de modo mais eficiente, transformando a Web em um grande banco de dados. Assim, a Web Semântica não é uma Web separada, mas uma extensão da atual, na qual a informação é publicada com um significado explícito e estruturado, permitindo melhor interação entre máquinas e pessoas (BERNERS-LEE; HENDLER; LASSILA, 2001).

Pode-se entender a Web Semântica como um conjunto de tecnologias e padrões que visam possibilitar que as máquinas entendam o significado, ou a semântica, das informações publicadas na Web e ontologia é uma delas (YU, 2011).

Uma ontologia representa formalmente um determinado domínio de conhecimento, definindo seus principais conceitos ou classes e relacionamentos que representam hierarquias (superclasses e subclasses). Outros relacionamentos também são encontrados nas ontologias, definidos por meio de propriedades que descrevem características ou atributos das classes, e também propriedades que relacionam termos com outros termos. Assim, uma ontologia codifica o conhecimento de um determinado domínio em um formato estruturado que possibilita a máquina entender esse conhecimento, viabilizando a Web Semântica (JACYNTHO, 2012). 
Segundo Noy e McGuinness (2001), uma ontologia representa um determinado domínio de conhecimento e consiste em: classes, que representam os principais conceitos do domínio; propriedades que representam relacionamentos e atributos das classes; axiomas, que são regras de restrição sobre o domínio e instâncias das classes.

Com os dados publicados na Web de forma estruturada e anotados semanticamente com ontologias, a máquina passa a compreender estes dados e, com o ferramental semântico descrito na ontologia, deduzir novos dados de maneira autônoma, gerando conhecimento.

\subsection{Sintomas, problemas e soluções}

A ontologia desenvolvida nesse trabalho tem como objetivo representar o domínio de sintomas, problemas e soluções. Por ser uma core ontology, ou seja, uma ontologia genérica para quaisquer tipos de problemas, há que se compreender como são percebidos esses elementos (sintomas, problemas e soluções) em diversos contextos.

Segundo o dicionário Michaelis (2009), um problema é uma questão levantada para inquirição, consideração, discussão, decisão ou solução, e acrescenta que um problema é um tema cuja solução ou decisão requer considerável meditação ou habilidade.

A definição de problema está intimamente relacionada com o contexto em que se aplica. Assim, no contexto da administração, um problema é indicado por alguma frustração, irritação, percepção de diferença entre a situação ideal e a real e perspectivas de prejuízos. Entende-se que um problema gera sempre uma decisão, ou seja, a partir da definição do problema, temos que buscar alternativas, ou soluções, para resolvê-lo (MAXIMIANO, 2004).

Ao se analisar o contexto de TI, o modelo ITIL v3 (CARTLIDGE et al., 2007) define um problema como uma causa não conhecida de um ou mais incidentes, ou seja, um incidente sinaliza um ou mais problemas. Um incidente pode ser considerado, dentro deste contexto, como uma interrupção não planejada ou redução na qualidade de um serviço de TI. Assim, com base nos incidentes (sinais da ocorrência de um problema) é identificado o problema que os causa. Após a identificação do problema, é aplicada uma solução, geralmente em forma de procedimentos (workflow).

Já na medicina, essa distinção fica bem clara, onde os problemas são identificados como doenças. Assim, um paciente reporta alguns sintomas, ou sinais, que indicam a existência de uma doença. O médico, a partir dos sintomas, identifica a doença que está causando os sintomas. Uma vez diagnosticada a doença, o paciente é submetido ao tratamento adequando. Um tratamento pode ser representado por uma sequência de passos como um workflow (MAYO CLINIC STAFF, 2013).

Com base nas abordagens de problemas nos contextos citados acima, conclui-se que um problema é algo ou alguém que não está no seu estado normal ou ideal. Quando algo ou alguém não se apresenta em seu 
estado ideal, sinais ou sintomas podem ocorrer evidenciando a existência do problema. Além disso, após a descoberta da causa dos sintomas, ou seja, a descoberta do problema em si, inicia-se o procedimento para solucionar o mesmo, onde, caso já exista uma solução previamente conhecida e catalogada, basta aplicá-la. Contudo, caso o problema seja desconhecido, é necessário estudar o problema visando o entendimento do mesmo para, posteriormente, elaborar e testar uma ou mais soluções. Um processo muitas vezes demorado e oneroso, cuja solução encontrada, obviamente, deve ser cuidadosamente catalogada para outras eventuais ocorrências futuras.

\section{IPO: ontologia para sintomas, problemas e soluções}

Para desenvolver a ontologia descrita nesse trabalho, foi utilizada a metodologia Ontology Development 101, descrita por Noy e McGuinness (2001). Essa metodologia foi escolhida pela simplicidade e iteratividade de suas etapas, a saber:

1) determine o domínio e o escopo da ontologia;

2) considere o reuso de ontologias existentes;

3) enumere os termos importantes da ontologia;

4) defina as classes e a hierarquia de classes;

5) defina as propriedades das classes;

6) defina as restrições;

7) crie instâncias.

Conforme mencionado anteriormente, a ontologia Issue Procedure Ontology (IPO) pretende representar o domínio de problemas, relacionando sintomas e soluções. Visando especificar o escopo e o domínio da ontologia, foram considerados inicialmente problemas comuns em alguns cenários motivacionais, dentre os quais, setor de suporte de TI de uma empresa (help desk), diagnóstico de doenças por um médico, identificação de defeitos na linha de produção de uma indústria e problemas no design de um software. Após analisá-los, foram elencadas algumas questões de competência para as quais a ontologia deveria prover as respostas. Estas questões servem para definir o escopo da ontologia, bem como avaliar sua expressividade. São elas:

QC1. Qual é a categoria de um problema/sintoma/solução?

QC2. Quais são sintomas de um problema?

QC3. Quais são as soluções de um problema?

QC4. Qual o causador do problema?

QC5. Qual o hospedeiro do problema?

QC6. Quem criou/registrou esse problema/sintoma/solução?

QC7. Quais ações (workflow) a serem tomadas para solucionar o problema?

QC8. Um problema causa outro problema? 
QC9. Um problema depende de outro problema?

QC10. Quais os possíveis problemas, a partir de um conjunto de sintomas?

QC11. Em quais possíveis classes (de problema) uma ocorrência no espaço/tempo pode ser classificada, a partir dos sintomas identificados?

A fim de se aplicar a diretriz de reuso de ontologias existentes, foi feito um levantamento das ontologias Linked Data relacionadas no indexador Linked Open Vocabularies $(\mathrm{LOV})^{2}$, onde verificou-se que nenhuma ontologia se propunha a descrever o domínio proposto com a expressividade necessária para responder as questões levantadas acima. Porém a IPO estende e/ou reusa termos de outras ontologias, a saber: Friend of a Friend (FOAF) (BRICKLEY; MILLER, 2014) - para descrição de pessoas e organizações; Dublin Core (DCMI USAGE BOARD, 2012) - para descrição de recursos gerais; Simple Knowledge Organization System (SKOS) (MILES; BECHHOFER, 2009) - para categorização de problemas por meio de um tesauro.

A ontologia foi construída usando-se a linguagem de ontologias Web Ontology Language (OWL 2.0), recomendada pelo W3C. Segundo Hitzler et al. (2012), OWL é uma linguagem projetada para representar o conhecimento sobre as "coisas", grupos de "coisas", e as relações entre as "coisas", com grande poder de expressividade.

Para a ontologia criada, foi adotado o prefixo ipo para representar o namespace http://purl.org/ipo/core\#. As especificações técnicas em RDF $^{3}$ de todos os termos da ontologia IPO foram publicadas na Web e podem ser encontradas no endereço http://purl.org/ipo/core.

O modelo conceitual da ontologia IPO pode ser visto na Figura 1, onde são apresentados seus conceitos (classes) e suas relações (propriedades), por meio de um diagrama de classes UML ${ }^{4}$.

\footnotetext{
${ }^{2}$ Disponível em: <http://lov.okfn.org/>. Acesso em: 14 maio 2015.

${ }^{3}$ Resource Description Framework (RDF) é o modelo de dados em grafo padrão da Web Semântica. Maiores detalhes podem ser obtidos em Cyganiak, Wood e Lanthaler $(2014$, p. 1).

${ }^{4}$ Disponível em: <http://www.uml.org/>. Acesso em: 15 maio 2015.
} 


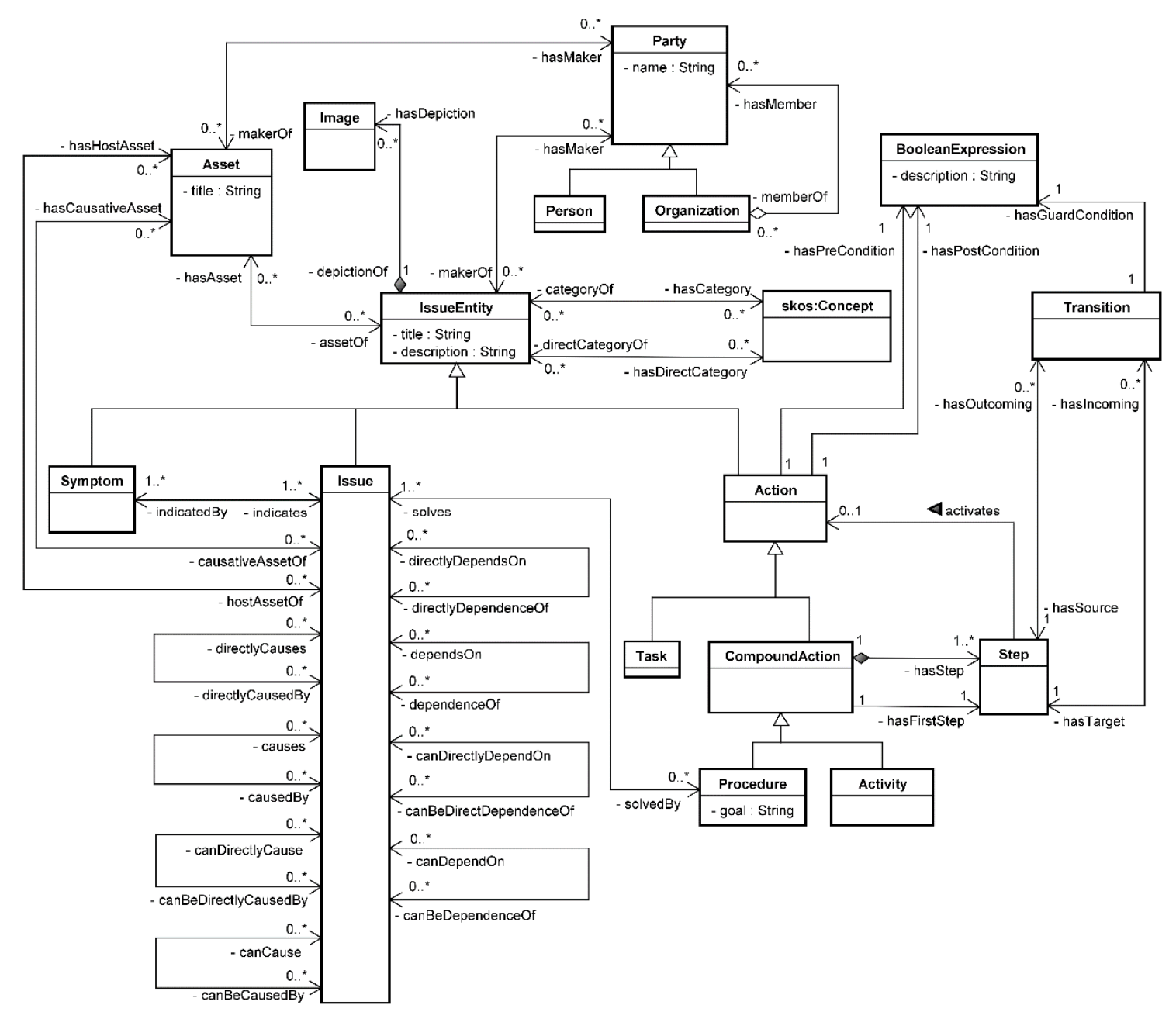

Figura 1 - Modelo conceitual da ontologia IPO

Fonte: Os autores (2015).

A seguir serão descritas as classes e as propriedades da ontologia IPO. Para tal, seguindo uma abordagem top-down, a ontologia foi dividida em quatro módulos, a saber:

\section{IssueEntity - Super Tipo Raiz}

Classes: IssueEntity, Asset, Image, skos:Concept.

Propriedades: categoryOf, hasCategory, directCategoryOf, hasDirectCategory, hasMaker, makerOf, hasAsset, assetOf, hasDepiction, depictionOf, title, description.

\section{Pessoas e Organizações}

Classes: Party, Person e Organization.

Propriedades: hasMember, memberOf, name.

\section{Problemas e Sintomas}

Classes: Symptom, Issue.

Propriedades: directlyCauses, directlyCausedBy, causes, causedBy, dependenceOf, dependsOn, directDependenceOf, directlyDependsOn, canDirectlyCause, canBeDirectlyCausedBy, 
canCause, canBeCausedBy, canBeDependenceOf, canDependOn, canBeDirectDependenceOf, canDirectlyDependOn, indicatedBy, indicates, hasCausativeAsset, causativeAssetOf, hasHostAsset, hostAssetOf.

\section{Problemas e Soluções}

Classes: Action, Task, CompoundAction, Procedure, Activity, Step, Transition, BooleanExpression.

Propriedades: solves, solvedBy, hasFirstStep, hasStep, activates, hasSource, hasTarget, hasIncoming, hasOutcoming, hasGuardCondition, hasPostCondition, hasPreCondition, goal.

\subsection{IssueEntity - super tipo raiz}

As classes e propriedades deste módulo visam descrever diversos relacionamentos que são comuns aos três principais termos da ontologia: Sintoma, Problema e Ação (Solução).

\section{Classe: IssueEntity}

Superconceito (supertipo) que reúne as características comuns aos três principais conceitos dentro do domínio da ontologia: Sintoma (Symptom), Problema (Issue) e Ação (Action).

\section{Classe: Asset}

Qualquer "coisa" de valor relacionada a uma IssueEntity ${ }^{5}$. Por exemplo, um problema (doença) diagnosticado em um paciente, pode ter um vírus e o paciente como Asset, pois o vírus é o agente causador do problema e o paciente é o hospedeiro no qual o problema se manifesta.

Um Asset pode ser qualquer "coisa": uma pessoa, um objeto, um relatório, um documento, etc.

\section{Classe: Image}

Um artefato que ilustra ou registra uma percepção visual.

Ela pode ser usada para ilustrar uma IssueEntity visando uma melhor compreensão dela.

\section{Classe: skos:Concept}

A ontologia IPO reusa a ontologia SKOS para definir esquemas de classificação, ou seja, um conjunto de categorias (ou conceitos) relacionadas (hierarquicamente e de outras formas) formando um tesauro, sob as quais instâncias da classe IssueEntity podem ser agrupadas.

A classe Concept da ontologia SKOS possui propriedades que permitem a criação de hierarquias de categorias (Concepts), permitindo

\footnotetext{
${ }^{5}$ Como a classe IssueEntity já foi descrita anteriormente, será utilizada o próprio nome da classe, em inglês, para citá-la no texto. Para os elementos ainda não descritos, serão citados em português e entre parênteses o nome da classe em inglês.
} 
ainda expressar transitividade entre as categorias. Essa abordagem deve ser utilizada como uma alternativa para classificação por subclasse de IssueEntity, quando se tratar de classificações não intrínsecas, apenas de agrupamento. Por exemplo, no domínio da medicina, podemos agrupar as doenças como doenças virais, doenças bacterianas, etc.

É importante ressaltar que existe outra forma de classificação, por meio da criação de subclasses de IssueEntity. O uso de subclasses leva a uma capacidade de inferência mais refinada e deve ser utilizado quando se tratar de classificação intrínseca de tipo/subtipo, onde novas classes, com novas restrições, precisam ser criadas para descrição de tipos de problema mais específicos de um contexto particular.

\section{Propriedades: hasCategory e categoryOf}

Uma IssueEntity pode ser agrupada em várias categorias, utilizando um esquema de classificação por meio de um tesauro de categorias (ou conceitos). A propriedade hasCategory relaciona uma IssueEntity com sua(s) categoria(s) (skos:Concept). categoryOf é propriedade inversa de hasCategory.

\section{Propriedades: hasDirectCategory e directCategoryOf}

hasDirectCategory é subpropriedade de hasCategory e indica uma categoria à qual a IssueEntity está diretamente relacionada. directCategoryOf é subpropriedade de categoryOf e propriedade inversa de hasDirectCategory.

\section{Propriedades: hasAsset e assetof}

hasAsset relaciona uma IssueEntity com um Asset. A propriedade assetof é propriedade inversa de hasAsset. assetof pode ser utilizada para facilitar a recuperação de registros de problemas relacionados ao Asset.

\section{Propriedades: hasDepiction e depictionof}

hasDepiction relaciona uma IssueEntity com uma Image, visando uma melhor descrição. depictionof é inversa de hasDepiction e é funcional, ou seja, uma Image está relacionada por essa propriedade com no máximo uma única IssueEntity.

\section{Propriedades: hasMaker e makerOf}

hasMaker associa uma IssueEntity com uma Pessoa ou Organização (Party) que a criou ou registrou. Essa propriedade também é utilizada pela classe Asset para relacionar seu fabricante, desenvolvedor, inventor, etc. makerOf é propriedade inversa de hasMaker.

\section{Propriedade: description}

Uma descrição textual que descreve algo com mais detalhes. 


\section{Propriedade: title}

Título (palavra ou frase) que resumidamente descreve algo.

\subsection{Pessoas e organizações}

Esse módulo provê termos para descrever pessoas e organizações, permitindo a representação de funcionários e colaboradores de empresas, definição de departamentos, entre outros.

\section{Classe: Party}

Superconceito (supertipo) comum aos conceitos Pessoa (Person) e Organização (Organization), e que assume um papel de agente (Party) dentro do domínio abordado. Um agente é uma pessoa ou organização atuante, ou seja, capaz de realizar algo.

\section{Classe: Person}

Esta classe representa pessoas. Um exemplo seria uma pessoa que trabalha em uma organização e produz algum Asset ou registra uma IssueEntity.

\section{Classe: Organization}

Representa um grupo de pessoas organizadas visando um objetivo em comum: social, comercial ou político. Por exemplo, uma empresa ou um departamento de uma empresa.

\section{Propriedades: hasMember e memberOf}

A propriedade hasMember relaciona uma Organization com seus membros, que são instâncias de Party, podendo ser Persons ou outras Organizations. Por exemplo, esta propriedade poderia ser usada para registrar que um funcionário é membro de um departamento. Uma outra abordagem é utilizar essa propriedade para relacionar duas organizações, representado, por exemplo, um departamento que é membro de sua empresa. memberOf é propriedade inversa de hasMember.

\section{Propriedade: name}

Nome para identificação de algo.

\subsection{Problemas e sintomas}

Esse é o módulo principal, pois contém termos que representam sintomas e problemas (Symptom, Issue) e os relacionam, permitindo que a máquina deduza, a partir dos sintomas, os possíveis problemas. 


\section{Classe: Issue}

Um problema ou questão a ser resolvida. Por exemplo, algo que não está operando normalmente ou um impedimento para realização de alguma tarefa.

Um Issue pode ser causa e/ou causado, direta ou indiretamente, por outro Issue, da mesma forma que o Issue A pode depender do Issue $\mathrm{B}$, precisando que o Issue B seja solucionado antes de solucionar A. O Issue pode ter um conjunto de ações (Procedure) que irá solucioná-lo e ainda, pode ser indicado por vários sintomas (Symptoms), onde um conjunto de Symptoms pode identificar um Issue.

\section{Classe: Symptom}

Representa um sintoma (sinal ou indicação) de um ou vários problemas (Issues). Algo que é percebido quando um problema ocorre.

\section{Propriedades: indicates e indicatedBy}

indicates relaciona um Symptom com um Issue que ele indica. indicatedBy é propriedade inversa de indicates.

Um Symptom pode indicar vários Issues, como por exemplo um sintoma de febre pode indicar diversas doenças.

\section{Propriedades: causes e causedBy}

causes registra que um Issue causa outro Issue de forma direta ou indireta. Esta propriedade expressa uma relação de causalidade entre Issues e possui a característica de transitividade, ou seja, se o Issue A causa o Issue B e o Issue B causa o Issue C, então o Issue A causa o Issue C. causedBy é propriedade inversa de causes.

\section{Propriedades: directlyCauses e directlyCausedBy}

directlyCauses é subpropriedade de causes e indica que um Issue causa outro Issue de forma direta. direcltyCausedBy é subpropriedade de causedBy e propriedade inversa de directlyCauses.

Por exemplo, considere um paciente que está com Pneumonia devido a uma Gripe mal curada, neste caso a Gripe foi causa direta da Pneumonia. Pelo axioma de subpropriedade, infere-se também que a Gripe foi a causa da Pneumonia.

\section{Propriedades: dependsOn e dependenceOf}

dependsOn relaciona um Issue com outro Issue do qual o primeiro depende direta ou indiretamente. Esta propriedade expressa uma relação de dependência entre Issues e possui a característica de transitividade, ou seja, se o Issue A depende do Issue B e o Issue B 
depende do Issue C, então o Issue A depende do Issue C. dependenceOf é propriedade inversa de dependsOn.

\section{Propriedades: directlyDependsOn e directDependenceOf}

direcltyDependsOn é subpropriedade de dependsOn e indica que um Issue depende diretamente de outro Issue. directDependenceOf é subpropriedade de dependenceof e propriedade inversa de directlyDependsOn.

Com base no exemplo exposto ao descrever a propriedade directlyCauses, a Pneumonia depende diretamente que a Gripe seja curada para, enfim, ser tratada. Pelo axioma de subpropriedade, deduz-se também que a Pneumonia depende da Gripe.

\section{Propriedade: canCause e canBeCausedBy}

canCause indica que um Issue pode causar outro Issue de forma direta ou indireta. Esta propriedade visa expressar uma relação de possível causalidade e possui a característica de transitividade, ou seja, se o Issue A pode causar o Issue B e o Issue B pode causar o Issue $\mathrm{C}$, então o Issue A pode causar o Issue C. canBeCausedBy é propriedade inversa de canCause.

\section{Propriedades: canDirectlyCause e canBeDirectlyCausedBy}

canDirectlyCause é subpropriedade de canCause e indica que um Issue pode causar outro Issue de forma direta. Esta propriedade visa expressar uma relação de possível causalidade, onde um Issue pode ser a causa direta de outro Issue. canBeDirectlyCausedBy é subpropriedade de canBeCausedBy e propriedade inversa de canDirectlyCause.

Por exemplo, a doença Gripe pode ser causa direta da Pneumonia, ou seja, em alguns casos a Gripe causa a Pneumonia e em outros casos não. Pelo axioma de subpropriedade, infere-se também que Gripe pode causar Pneumonia.

\section{Propriedades: canDependOn e canBeDependenceOf}

canDependOn indica que um Issue pode depender de outro Issue direta ou indiretamente. Esta propriedade expressa uma relação de possível dependência entre Issues e possui a característica de transitividade, ou seja, se o Issue A pode depender do Issue B e o Issue $\mathrm{B}$ pode depender do Issue $\mathrm{C}$, então o Issue A pode depender do Issue C. canBeDependenceOf é propriedade inversa de canDependOn.

\section{Propriedades:}

\section{canDirectlyDependOn e canBeDirectDependenceOf}

canDirectlyDependOn é subpropriedade de canDependOn e indica que um Issue pode depender diretamente de outro Issue. 
canBeDirectDependenceOf é subpropriedade de canBeDependenceOf e propriedade inversa de canDirectlyDependOn.

Com base no exemplo exposto ao descrever a propriedade canDirectlyCause, a Pneumonia pode depender, de forma direta, que a Gripe seja curada para, enfim, ser tratada. Pelo axioma de subpropriedade, Pneumonia pode depender de Gripe.

\section{Propriedades: hasCausativeAsset e causativeAssetOf}

hasCausative relaciona um Issue com algum agente (Asset) causador deste Issue. causativeAssetOf é propriedade inversa de hasCausativeAsset.

Por exemplo, uma doença relacionada com seu o agente causador (um vírus, bactéria, etc.).

\section{Propriedades: hasHostAsset e hostAssetOf}

hasHostAsset relaciona um Issue com hospedeiro (Asset) no qual este Issue ocorreu. hostAssetOf é propriedade inversa de hasHostAsset.

Por exemplo, uma doença relacionada com a pessoa doente, ou seja, a pessoa é o hospedeiro da doença.

\subsection{Problemas e soluções}

As classes e propriedades desse módulo relacionam a classe Issue com a classe Procedure que representa uma possível solução para o problema. Ainda provê meios para delinear uma solução por meio de um workflow.

\section{Classe: Action}

Representa uma ação a ser realizada para resolver o problema. Uma Action pode ser apenas uma ação primitiva ou tarefa (Task) ou um conjunto de ações (CompoundAction).

\section{Classe: Task}

Uma ação elementar e atômica, que executa algo simples.

\section{Classe: CompoundAction}

Uma ação composta por várias outras ações. Uma CompoundAction pode ter o objetivo de solucionar um ou mais Issues, representando um procedimento (Procedure) ou não ter um objetivo explícito, apenas ser um grupo de Actions a ser reusado, se comportando como uma atividade (Activity). Uma CompoundAction possui um ou mais passos de execução (Steps) que ativam uma Action (Task ou outra CompoundAction), permitindo assim, que uma CompoundAction reuse outras.

Uma CompoundAction pode ser utilizada para criar uma estrutura de workflow, visando uma melhor estruturação das ações que a compõem. 


\section{Classe: Procedure}

Uma sequência de passos (Steps) com objetivo explícito que após executados solucionam um ou mais Issues.

\section{Classe: Activity}

Um conjunto de passos (Steps) que realizam uma atividade, porém não tem um objetivo explícito e, portanto, não visa a solução de um determinado Issue. Na verdade, trata-se de um agrupamento de ações visando reuso.

\section{Classe: Step}

Um passo ou etapa a ser realizada dentro de uma CompoundAction. Todo Step possui uma Action a ser executada e uma transição para outro Step a ser efetuada após finalizada a execução da Action. Com os Steps é possível estabelecer uma ordem para a execução das Actions, pois cada Step possui uma transição (Transition) que estabelece o Step de origem e de destino. Uma vez que uma CompoundAction possui um Step inicial (hasFirstStep), a partir desse Step, é possível executar todos os outros Steps que compõe a CompoundAction, seguindo a transição entre eles.

\section{Classe: Transition}

Transition representa a transição entre dois Steps. Cada Transition tem um Step de origem (source) e um Step de destino (target). Uma Transition possui uma condição de guarda que especifica uma expressão booleana (BooleanExpression) que tem que ser verdadeira para que a transição ocorra.

Através da condição de guarda, pode-se implementar, de maneira simplificada, um workflow, estabelecendo estruturas de decisão, repetição, escolha, etc.

\section{Classe: BooleanExpression}

Uma expressão lógica cujo valor é verdadeiro ou falso, visando validar uma transição entre dois Steps, ou servir com uma précondição para a execução de uma Action ou ainda, servir como uma pós-condição que valide a execução de uma Action.

\section{Propriedade: hasPreCondition}

Indica uma pré-condição para que a Action seja executada. Uma pré-condição representa um pré-requisito definido por uma expressão booleana (BooleanExpression) para execução da Action, sem o qual não é garantida a execução correta da mesma. 


\section{Propriedade: hasPostCondition}

Indica uma pós-condição (efeito) definida por uma expressão booleana (BooleanExpression) que será atingida após a execução da Action, desde que a pré-condição tenha sido respeitada.

\section{Propriedade: hasStep}

Indica um passo de execução (Step) que compõe a CompoundAction.

\section{Propriedade: hasFirstStep}

Uma CompoundAction possui um ou mais passos (Steps), assim essa propriedade indica o primeiro passo pelo qual a execução da CompoundAction é iniciada.

\section{Propriedade: goal}

Descrição do objetivo que se deseja alcançar após a execução do Procedure.

\section{Propriedades: solves e solvedBy}

solves relaciona a Procedure com o Issue que a ela soluciona. solvedBy é propriedade inversa de solves.

\section{Propriedade: activates}

Indica a ação a ser ativada por um Step.

\section{Propriedades: hasIncoming e hasTarget}

hasIncoming indica as transições (Transitions) de entrada do Step, ou seja, as que iniciam a execução deste Step. hasTarget é propriedade inversa de hasIncoming, indicando o Step destino da Transition. hasTarget é também uma propriedade funcional, uma vez que uma Transition tem um único Step destino.

\section{Propriedades: hasOutcoming e hasSource}

hasOutcoming indica as transições (Transitions) de saída do Step, ou seja, as que ocorrem após a execução deste Step e que acionam os próximos Steps a serem executados. hasSource é propriedade inversa de hasOutcoming, indicando o Step de origem da Transition. hasSource é também um propriedade funcional, uma vez que uma Transition tem um único Step de origem.

\section{Propriedade: hasGuardCondition}

Indica uma condição booleana (BooleanExpression) para que uma Transition ocorra.

Por exemplo, uma Transition pode ter como guardCondition que o Step de origem seja executado 10 vezes. Assim, enquanto este Step não for executado 10 vezes, não será iniciando o Step destino. Este exemplo ilustra uma estrutura de repetição dentro de um workflow. 


\section{Exemplo de instanciação da ontologia}

Para demonstrar o uso da ontologia IPO, a Figura 2 ilustra o mapeamento de instâncias (RDF) para classes/propriedades da ontologia (OWL), para o domínio de problemas médicos (doenças), onde foi abordada a Gripe como exemplo de instância da classe Issue. É demonstrado o relacionamento entre o problema Gripe com o sintoma Febre, instância da classe Symptom. O problema Gripe também é relacionado com um possível tratamento, instância da classe Procedure. Outro relacionamento representado é o vírus Influenza, instância da classe Asset, que possui uma relação de causador da Gripe. Além disso, é demonstrado um relacionamento entre o problema Gripe com seu hospedeiro, no caso, o Ser Humano, também instância da classe Asset. Como forma de organização, a Gripe foi agrupada na categoria Doenças Virais, instância da classe skos:Concept. Para demonstrar a dependência entre problemas, foi adicionado o problema Pneumonia, onde a Gripe pode causar Pneumonia e Pneumonia pode depender que a Gripe seja solucionada para, então, ser tratada. Os relacionamentos representados na cor verde são inferidos automaticamente pela máquina com base no axioma de subpropriedade. Para todos os outros relacionamentos, com base no axioma de propriedade inversa, os respectivos relacionamentos inversos também seriam inferidos, mas não foram ilustrados para não sobrecarregar a Figura 2. 


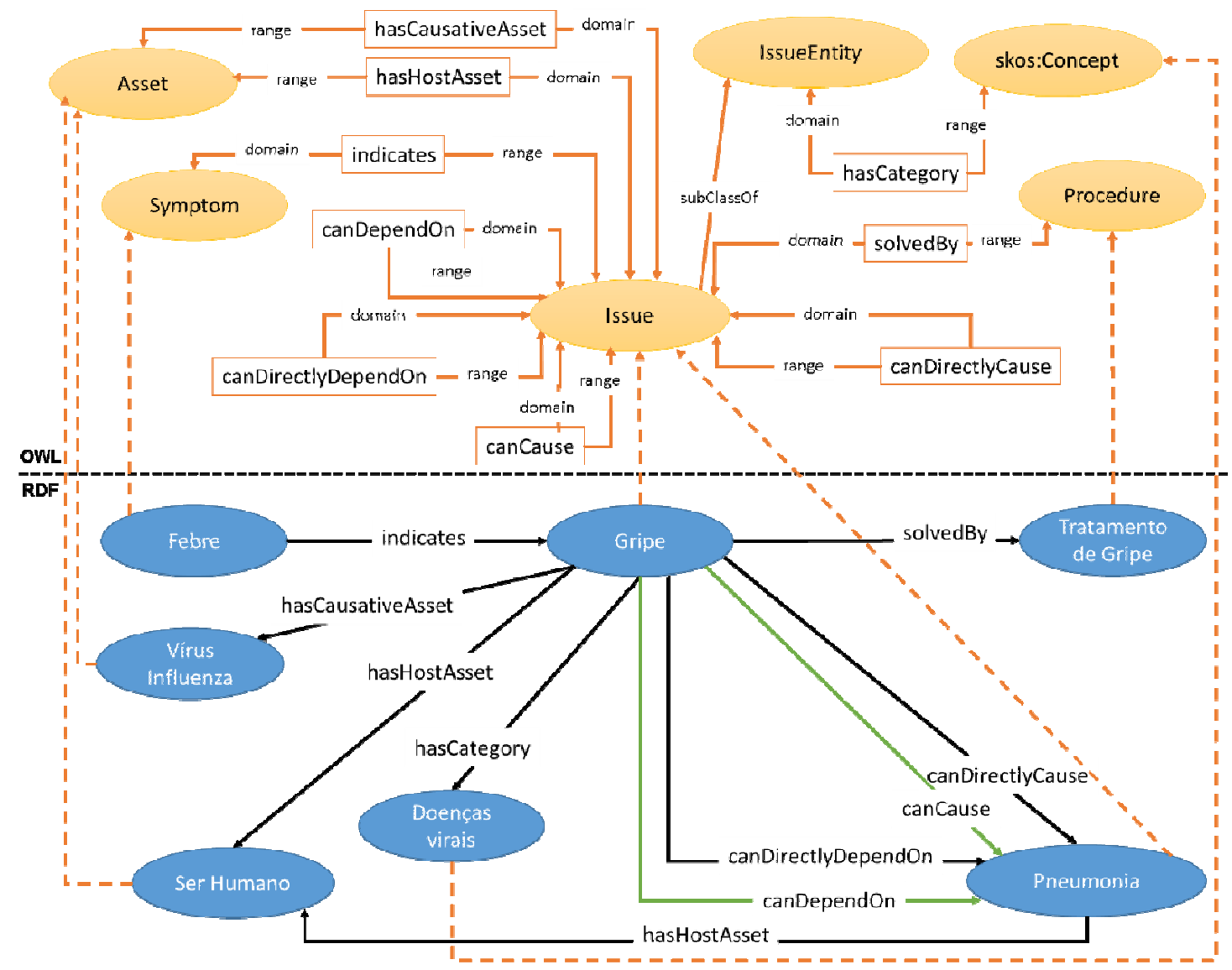

Figura 2 - Mapeamento entre dados RDF e a ontologia IPO Fonte: Os autores (2015).

\section{Exemplo de instanciação de procedimento (Procedure)}

Como já foi dito anteriormente, um procedimento é representado por um workflow, contendo os passos a serem executados. A ontologia IPO fornece meios para representar, semanticamente, fluxogramas instanciando a classe Procedure. A Figura 3 fornece um exemplo de um procedimento para tratamento do problema Pneumonia representado por um fluxograma. 


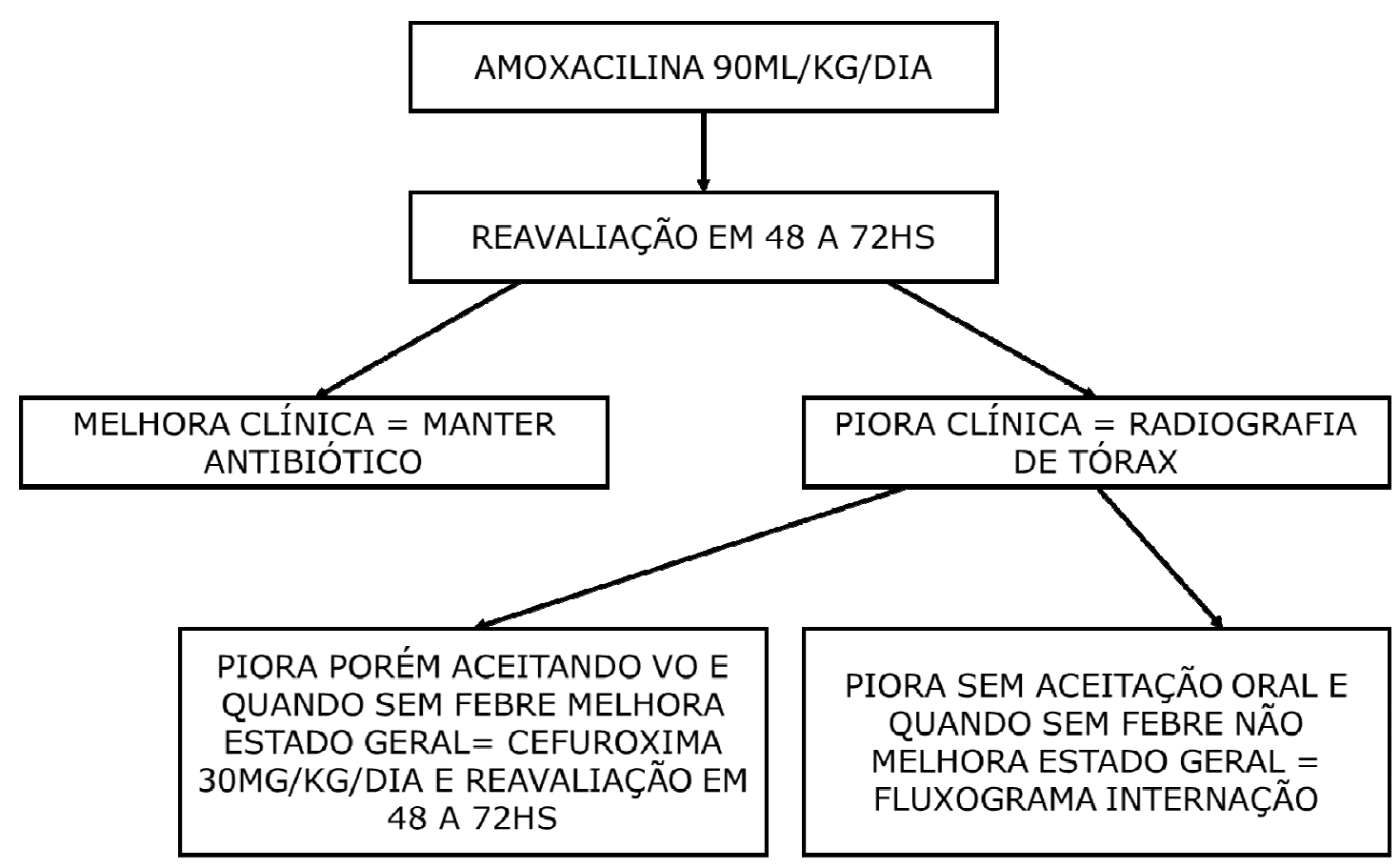

Figura 3 - Algoritmo de tratamento da Pneumonia

Fonte: MAYO CLINIC STAFF (2013).

A ontologia IPO determina que um Procedure possui um objetivo (goal) a ser alcançado no final de sua execução. Além disso, um Procedure possui (hasStep) um ou mais passos (Step), a serem executados (Step). Cada Step ativa (activates) uma ação (Action) que deve ser executada.

Uma Action é superclasse de Task, que representa uma tarefa simples e atômica, e de CompoundAction, que representa uma ação composta por diversas outras ações, simples ou compostas.

Após executar a Action de um determinado Step, deve-se passar para o próximo Step, ocorrendo assim, uma transição entre etapas (Transition). Cada Transition, porém, possui uma condição de guarda (hasGuardCondition) que consiste em uma expressão lógica (BooleanExpression) que deve ser atendida para que a Transition ocorra. Essas condições de guarda possibilitam que estruturas mais complexas de fluxograma, como condicionais e repetições, sejam construídas.

Para exemplificar uma instanciação da classe Procedure, a Figura 4 traz o procedimento de tratamento da Pneumonia, que pode ser visto na Figura 3, instanciado com a ontologia IPO. É importante notar que o ipom:Step5 ativa um novo procedimento, ou seja, um Procedure pode fazer uso de outro Procedure, o que é bastante útil para o reuso de procedimentos ou tratamentos. 


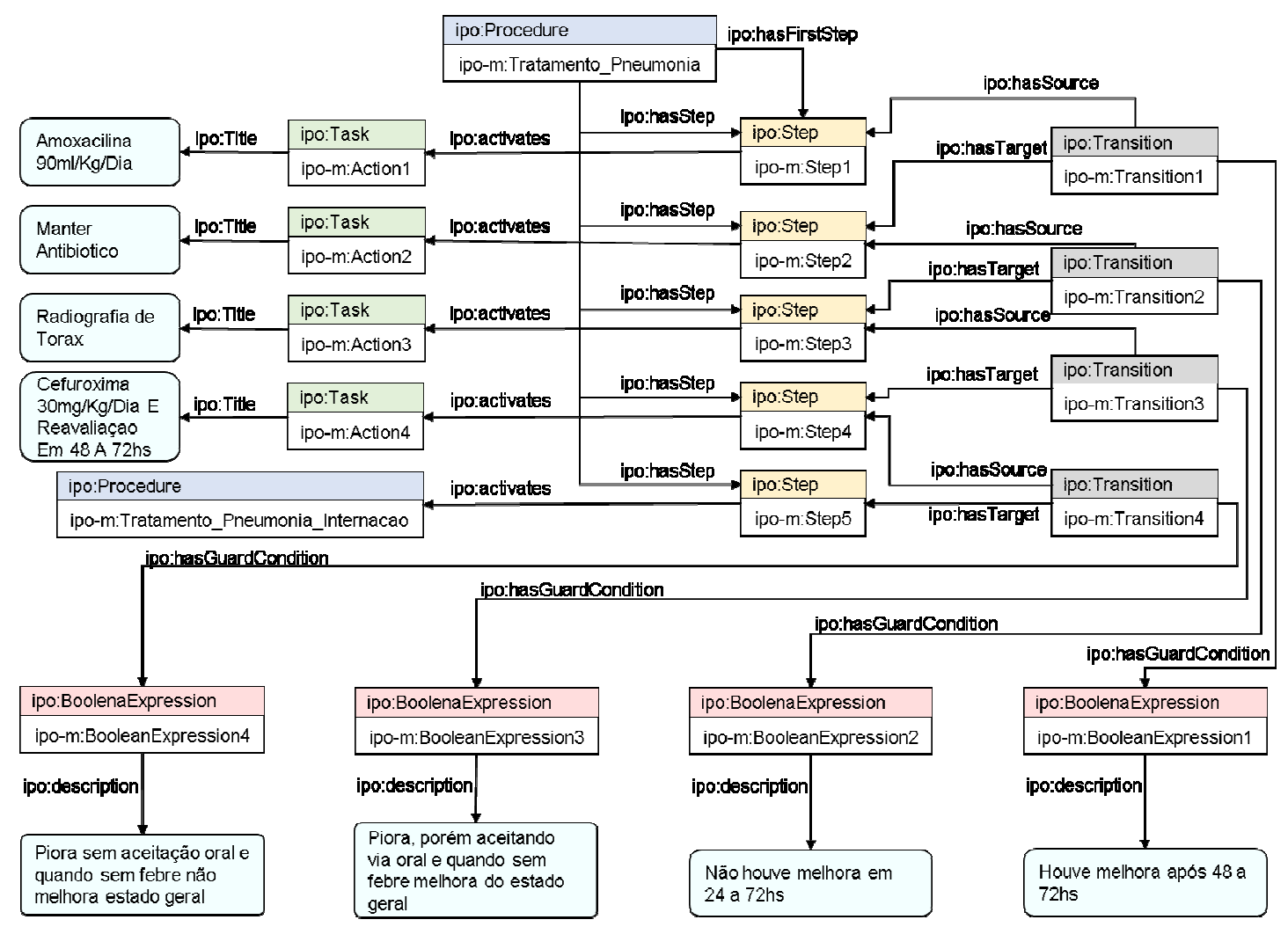

Figura 4 - Instanciação do algoritmo de tratamento da Pneumonia Fonte: Os autores (2015).

\section{Avaliação da ontologia proposta}

A avaliação é uma etapa importante no processo de criação de uma ontologia. Para realizar essa validação, se faz necessário verificar se a ontologia é expressiva o suficiente para prover respostas para as questões de competência levantadas anteriormente e verificar se ela consegue representar todos os dados pertinentes ao domínio.

Por se tratar de uma ontologia extensível (core ontology), a utilização deve ser feita a partir de especializações para um domínio mais específico, como por exemplo, o domínio de problemas e soluções médicas.

Como dito anteriormente, existem duas formas de classificação de uma IssueEntity: (1) uma classificação não intrínseca, ou seja, uma classificação visando um simples agrupamento de IssueEntities, onde a ontologia IPO provê a utilização da propriedade hasCategory relacionando com skos:Concept, possibilitando a criação de esquemas de categorias e subcategorias; (2) uma classificação intrínseca a um IssueEntity, de modo que essa classificação implica na criação de subclasses de IssueEntity, com restrições específicas. Por exemplo, a classe Gripe, sendo definida por uma restrição específica que é estar relacionado a pelo menos um dos sintomas Febre ou Coriza (instância da classe Symptom). Desta forma, ocorrências podem ser classificadas, com base na descrição de seus sintomas, como sendo uma instância da nova classe Gripe. 
A seguir, para cada questão de competência, será demonstrado os meios oferecidos pela ontologia IPO que permitem responder a cada questão. Foi adotada a instanciação exposta na seção 4 para exemplificação das questões.

\section{QC1. Qual é a categoria de um problema/sintoma/solução?}

A propriedade hasCategory relaciona uma IssueEntity (classe genérica para Symptom, Issue e Action) com a classe skos:Concept. Assim, através dessa propriedade é possível obter a categoria (ou categorias) à qual a IssueEntity está relacionada. Como exemplo, se obteria a categoria Doenças Virais para o problema Gripe através da propriedade hasCategory. É importante notar que a IPO ainda provê a propriedade inversa isCategoryof que relaciona a classe skos:Concept com uma IssueEntity, o que permite se obter todas as IssueEntities de uma determinada categoria.

\section{QC2. Quais são sintomas de um problema?}

A propriedade indicatedBy relaciona um Issue com a classe Symptom. Dessa forma, essa propriedade permite obter o(s) sintoma(s) que sinaliza(m) o Issue.

A ontologia IPO provê ainda a propriedade inversa indicates que relaciona um Symptom com um Issue, onde, através desta, se pode obter todos os Issues indicados por um dado sintoma. Por exemplo, o sintoma Febre indica Gripe.

\section{QC3. Quais são as soluções de um problema?}

Para se obter essa resposta, pode ser utilizada a propriedade solvedBy, que relaciona um Issue com a classe Procedure. Portanto, essa propriedade permite obter o(s) procedimento(s) que soluciona(m) o Issue.

Para se obter todos os problemas solucionados por um Procedure, pode ser fazer uso da propriedade inversa solves. Como pode ser visto no caso de a Gripe ser solucionada pelo procedimento Tratamento de Gripe.

\section{QC4. Qual o causador do problema?}

Uma informação importante sobre um problema é o seu causador. Para tal, a IPO fornece a propriedade hasCausativeAsset que relaciona um Issue com um Asset. Assim, através dessa propriedade é possível obter o causador de um dado problema.

Também é possível saber todos os problemas causados por um Asset, por meio da propriedade inversa causativeAssetOf. Por exemplo, a Gripe tem como agente causador o vírus Influenza.

\section{QC5. Qual o hospedeiro do problema?}

A propriedade hasHostAsset pode ser utilizada para relacionar um Issue com o seu hospedeiro (Asset), ou seja, relacionar um problema com a "coisa" na qual ele ocorre. 
Também é possível saber todos os problemas que ocorreu em um determinado Asset, por meio da propriedade inversa hostAssetOf. Por exemplo, Gripe tem como hospedeiro o Ser Humano.

QC6. Quem criou/registrou esse problema/sintoma/solução?

A propriedade hasMaker relaciona um IssueEntity com a classe Party (Person ou Organization). Assim, através dessa propriedade se pode obter a pessoa ou organização que criou ou registrou uma determinada IssueEntity. É importante notar que a IPO ainda oferece a propriedade inversa makerOf que relaciona a classe Party com uma IssueEntity, o que permite se obter todas as IssueEntities criadas por uma pessoa ou organização. Não consta no exemplo de instanciação, porém, um médico poderia estar relacionado com o registro do problema Gripe por meio da propriedade hasMaker.

\section{QC7. Quais ações (workflow) a serem tomadas para solucionar o problema?}

Com já mencionado, Procedure é a classe que representa a solução de um problema e é composta por um ou mais Steps. Cada Step ativa uma ação a ser executada. Além disso, o Step possui uma Transition de saída, que irá promover a transição para o próximo Step do Procedure.

Sendo assim, para se obter todas as ações a serem sequencialmente executadas, primeiramente deve se obter o primeiro Step do Procedure, através da propriedade hasFirstStep. A partir desse primeiro Step, é possível, através da propriedade hasOutcomming, obter a Transition que irá acionar o próximo Step.

A propriedade hasTarget, que relaciona uma Tansition com um Step, indica o Step destino da transição.

Através das classes e propriedades aqui mencionadas, é possível obter todos os Steps, ordenados, que deverão ser executados.

Para se obter as ações referente a cada Step, basta fazer uso da propriedade activates, que relaciona o Step com a ação a ser executada.

\section{QC8. Um problema causa outro problema?}

A propriedade causes relaciona a classe Issue com ela própria. Esse relacionamento expressa que um Issue causa outro Issue. A propriedade directlycauses também pode expressar um relacionamento de causalidade, porém com a característica de ser uma causalidade direta, ou seja, indicando que um Issue causa diretamente outro.

Assim, a propriedade causes expressa que o Issue causa outro Issue de maneira direta ou indireta.

Com essas propriedades é possível saber se um problema causa outro, direta ou indiretamente. 


\section{QC9. Um problema depende de outro problema?}

A propriedade dependsOn relaciona a classe Issue com ela própria. Esse relacionamento expressa que um Issue depende de outro Issue. A propriedade directlyDependsOn também pode expressar um relacionamento de dependência, porém com a característica de ser uma dependência direta, ou seja, indicando que um Issue depende diretamente de outro.

Assim, a propriedade dependsOn expressa que o Issue depende de outro Issue de maneira direta ou indireta.

Com essas propriedades é possível saber se um problema depende de outro, direta ou indiretamente.

\section{QC10. Quais os possíveis problemas, a partir de um conjunto de sintomas?}

Fortemente relacionada à questão QC2, esta questão pode ser respondida por meio da propriedade indicates que relaciona um Symptom com o Issue que ele indica. Dado um conjunto de Symptoms, é possível obter todos os Issues indicados por estes Symptoms. Para melhorar ainda mais, os Issues retornados podem ser ordenados pela quantidade de Symptoms presentes no referido conjunto, obtendo-se, assim, uma lista de Issues ordenados do mais provável para o menos provável, usando-se para tal a propriedade inversa indicatedBy, que relaciona o Issue com seus Symptons.

QC11. Em quais possíveis classes (de problema) uma ocorrência no espaço/tempo pode ser classificada, a partir dos sintomas identificados?

A classe Issue tem como objetivo descrever problemas. Para que possamos registrar semanticamente as ocorrências no espaço/tempo de problemas, faz-se necessário criar subclasses da classe Issue que representem os problemas e essas subclasses terão como instancias as ocorrências.

Diante disso, visando permitir a classificação automática de ocorrências de problemas a partir de seus sintomas, a título de exemplo de classificação intrínseca em um domínio específico, foi criada uma subclasse da classe Issue denominada Gripe, definida como equivalente ao conjunto de recursos que apresentam pelo menos um dos sintomas (instâncias da classe Symptom): Febre ou Coriza. Em outras palavras, qualquer ocorrência que apresente pelo menos um destes dois sintomas, a máquina, automaticamente, a classificará como sendo uma instância da classe Gripe. A Figura 5 demonstra esse exemplo.

As relações na cor verde são obtidas automaticamente quando a máquina classifica a ocorrência como instância da classe Gripe. Perceba que o ser humano apenas precisa informar os sintomas (relacionamentos na cor preta) e a máquina decide, com base na ontologia, quais possíveis doenças se aplica àquela ocorrência. 


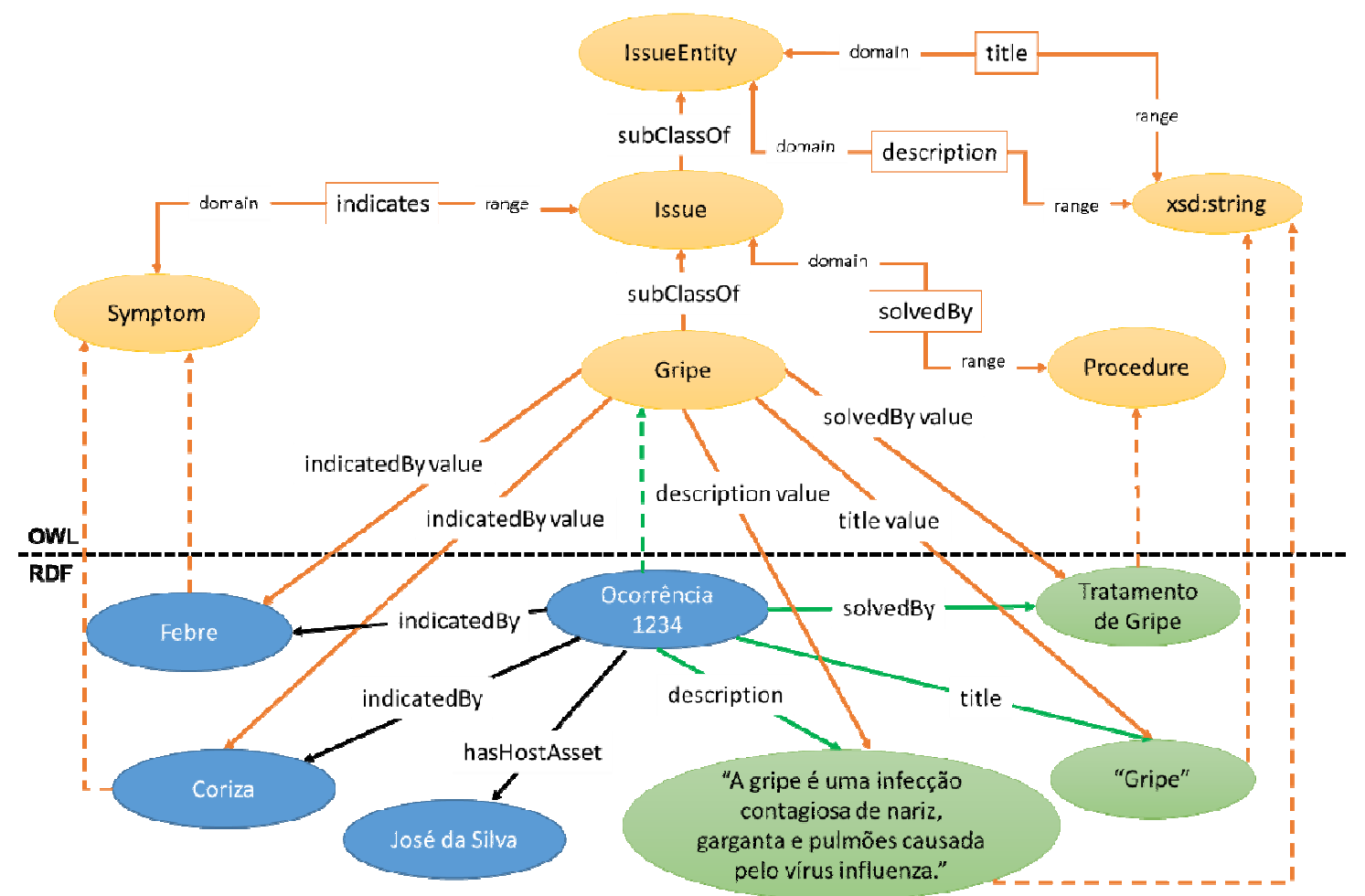

Figura 5 - Exemplo de instanciação com a subclasse Gripe

Fonte: Os autores (2015).

\section{Considerações finais e trabalhos futuros}

A Issue Procedure Ontology (IPO) tem o objetivo de representar o domínio de problemas, relacionando sintomas, problemas e soluções. Para sua construção, foi elaborado um conjunto de questões de competência visando definir algumas perguntas-chave que, depois de finalizada, a ontologia deveria prover meios para responder. Assim a ontologia IPO contém 16 classes, 48 propriedades e 2 indivíduos implementados utilizando a linguagem OWL 2.0 e todos os termos foram apresentados na seção 3.

A fim de se avaliar se a ontologia atende às questões de competência, foram apresentados exemplos realistas de instanciação evidenciando o poder de expressividade da ontologia.

A ontologia fornece os mecanismos necessários para que a máquina seja capaz de identificar os possíveis problemas a partir de sintomas, bem como sugerir possíveis soluções a partir dos problemas identificados. Diferentemente de um sistema de informação, onde a máquina fornece informações para que o ser humano resolva o problema, a IPO representa um passo fundamental em direção a construção de sistemas de conhecimento, onde a máquina forneça não apenas informações, mas também forneça possíveis soluções, de forma autônoma, para um problema em questão.

Como a ontologia IPO é uma ontologia extensível (core ontology), para se aplicar em contexto real de forma mais fidedigna, é recomendado especializá-la para se obter uma maior adequação ao domínio a ser 
abordado. No decorrer deste artigo, foram expostos diversos exemplos relacionados com o domínio problemas médicos e, paralelamente a ontologia, está sendo desenvolvido um estudo de caso realista dentro deste domínio, abordando algumas doenças, com seus sintomas e tratamentos, onde a ontologia IPO é estendida por subclasses descrevendo especificamente cada uma destas doenças.

Como importante trabalho futuro, esta ontologia precisa ser inserida em alguns contextos reais, sendo utilizada por um período considerável de tempo para que seja paulatinamente ajustada às necessidades dos usuários. Um processo contínuo de aperfeiçoamento peculiar a construção de ontologias.

\section{Referências}

BERNERS-LEE, T.; HENDLER, J.; LASSILA, O. The semantic web. Scientific American, v. 284, n. 5, p. 28-37, 2001.

BRICKLEY, D.; MILLER, L. FOAF vocabulary specification 0.99. 2014. Disponível em: <http://xmlns.com/foaf/spec/>. Acesso em: 11 maio 2015.

CARTLIDGE, A. et al. An introductory overview of ITIL\& V3. $1^{a}$ ed. Wokingham: It Service Management Forum Limited, 2007. 56 p.

Disponível em:

<http://www.itsmf.org.rs/sites/default/files/itSMF\%20ITIL\%20V3\%20Intr oduction\%200verview.pdf>. Acesso em: 20 maio 2015. 978-0-95512458-7.

CYGANIAK, R.; WOOD, D.; LANTHALER, M. RDF 1.1 concepts and abstract syntax. W3C Recommendation, 2014. Disponível em:

<http://www.w3.org/TR/rdf11-concepts/>. Acesso em: 11 maio 2015.

DCMI USAGE BOARD. DCMI metadata terms. 2012. Disponível em: $<$ http://dublincore.org/documents/dcmi-terms/>. Acesso em: 11 maio 2015.

HITZLER, P. et al. OWL 2 Web Ontology Language Primer. 2a ed. W3C Recommendation, 2012. Disponível em:

<http://www.w3.org/TR/2012/REC-owl2-primer-20121211/>. Acesso em: 05 maio 2015.

JACYNTHO, M. D. DE A. Um modelo de bloqueio multigranular para RDF. Rio de janeiro: Pontifícia Universidade Católica do Rio de Janeiro; Departamento de Informática, 2012.

LICHTNOW, D.; DE OLIVEIRA, J. P. M. Relato e Considerações sobre o Desenvolvimento de uma Ontologia para Avaliação de Sites da Área de Saúde. Cadernos de Informática, v. 4, n. 1, p. 7-46, 2009.

MAGALHÃES, I. L. ; PINHEIRO, W. B. Gerenciamento de serviços de TI na prática: uma abordagem com base na Itil. São Paulo: Novatec, 2007.

MAXIMIANO, A. C. A. Introdução à administração. São Paulo: Atlas, 2004. 
MAYO CLINIC STAFF. Pneumonia. 2013. Disponível em: <http://www.mayoclinic.org/diseasesconditions/pneumonia/basics/definition/con-20020032>. Acesso em: 10 jan. 2015.

MICHAELIS, H. Michaelis: dicionário brasileiro da língua portuguesa. 2015. Disponível em: <http://michaelis.uol.com.br/>. Acesso em: 30 mar. 2015.

MILES, A.; BECHHOFER, S. SKOS Simple Knowledge Organization System Namespace Document-HTML. W3C Recommendation. 2009. Disponível em: <http://www.w3.org/2009/08/skos-reference/skos.html>. Acesso em: 11 maio 2015.

NOY, N. F.; MCGUINNESS, D. L. Ontology development 101: a guide to creating your first ontology. 2001. Stantford Medical Informatics Technical Report, SMI-2001-0880. Disponível em:

<http://protege.stanford.edu/publications/ontology_development/ontolog y101.pdf>. Acesso em: 24 abril 2015.

PEREIRA, J. R.; DE SOUZA, M. A.; DA COSTA, H. R. Gerenciamento de problema: uma abordagem com base na Itil. Pensar Tecnologia, v. 1, n. 2, p. 23-36, 2012.

PICKLER, M. E. V. Web semântica: ontologias como ferramentas de representação do conhecimento. Perspectivas em Ciência da Informação, v. 12 , n. 1, p. 65-83, 2007.

REZENDE, D. A. Tecnologia da informação integrada à inteligência empresarial. São Paulo: Atlas, 2002.

YU, L. A developer's guide to the semantic web. Berlin: Springer, 2011. 